\title{
Update on Gastric Cancer Epidemiology and Risk Factors
}

\author{
Nada Lahmidani ${ }^{*}$, Mounia El Yousf ${ }^{1}$, Nourdine Aqodad', Dafr Allah Benajah', \\ Mohammed El Abkari' ${ }^{1}$, Adil Ibrahimi' ${ }^{1}$, Adil Najdi' ${ }^{2}$, Zineb Benbrahim ${ }^{3}$, Naoufal Mellas ${ }^{3}$ \\ ${ }^{1}$ Department of Gastroenterology, University Hospital Hassan II, School of Medicine and Pharmacy of Fez, \\ Sidi Mohammed Ben Abdallah University, Fez, Morocco \\ ${ }^{2}$ Department of Epidemiology and Biostatistics, School of Medicine and Pharmacy of Tangier, Tangier, Morocco \\ ${ }^{3}$ Department of Medical Oncology, University Hospital Hassan II, School of Medicine and Pharmacy of Fez, \\ Sidi Mohammed Ben Abdallah University, Fez, Morocco \\ Email: *nadalahmidani1982@gmail.com
}

How to cite this paper: Lahmidani, N., El Yousf, M., Aqodad, N., Benajah, D.A., El Abkari, M., Ibrahimi, A., Najdi, A., Benbrahim, Z. and Mellas, N. (2018) Update on Gastric Cancer Epidemiology and Risk Factors. Journal of Cancer Therapy, 9, 242-254.

https://doi.org/10.4236/jct.2018.93021

Received: December 26, 2017

Accepted: March 11, 2018

Published: March 14, 2018

Copyright (C) 2018 by authors and Scientific Research Publishing Inc. This work is licensed under the Creative Commons Attribution International License (CC BY 4.0).

http://creativecommons.org/licenses/by/4.0/

\begin{abstract}
Gastric adenocarcinoma is the most common gastric tumor. It is the fifth most common cancer worldwide after lung cancer, breast, colo rectal and prostate cancer. Long considered enigmatic, its epidemiology has changed over time. In fact, the incidence of distal gastric cancer has declined while that of the cardia was stable or increased. This cancer is multifactorial but reducing the incidence of distal cancer is particularly attributed to advances in the treatment of $H$. pylori infection. In this review, we analyzed the available data concerning the changing epidemiology of gastric cancer and the main risk factors. The incidence of distal cancer is definitely declining due to the control of Helicobacter pylori infection. Proximal gastric cancer and cardia cancer is particularly due to smoking, overweight.
\end{abstract}

\section{Keywords}

Adenocarcinoma, Gastric, Incidence, Risk Factors, Helicobacter pylori

\section{Introduction}

Gastric adenocarcinoma is the most common gastric tumor that develops from the gastric epithelium. It accounts for $95 \%$ of gastric cancer. Its incidence is high in the eastern countries and low in Africa which has made its epidemiology enigmatic. In fact, the incidence differs depending of the proximal or distal localization of the tumor. Despite its declining epidemiology due to identification of certain risk factors such as $H$. pylori and other dietary and environmental risks, it remains one of the leading causes of cancer mortality probably because of the 
late diagnosing stage. In this article, we will review the changing epidemiology of gastric adenocarcicoma and the current knowledge about its risk factors to understand its trend and the necessary measures to prevent it.

\section{Descriptive Epidemiology}

\subsection{Cancer Statistics}

\subsubsection{Incidence-Death Rates}

According to Globocan 2012, almost one million new cases of stomach cancer were estimated to have occurred in 2012 (952,000 cases, $6.8 \%$ of the total) [1] [2]. It's considered the fifth most common cancer in the world, after cancers of the lung, breast, colorectum and prostate. More than $70 \%$ of cases $(677,000$ cases) occur in developing countries (456,000 in men, 221,000 in women). Worldwide, stomach cancer is more common in Japan, China, Southern and Eastern Europe, and South and Central America. This disease is less common in Northern and Western Africa, South Central Asia, and North America [3]. Age-standardized incidence rates are about twice as high in men as in women, ranging from 3.3 in Western Africa to 35.4 in Eastern Asia for men, and from 2.6 in Western Africa to 13.8 in Eastern Asia for women [3] (Figure 1).

In the US in 2011, there were an estimated 74,035 people living with stomach cancer [4] and the estimated age adjusted incidence rate is about 22.22 cases/100,000 population in the year 2014 ( $1.3 \%$ of all new cancer cases in the US) making it the 16th common cancer in the US. The lifetime risk of developing gastric cancer is approximately $0.9 \%$.

In the US, stomach cancer is most frequently diagnosed among people aged $65-74$ and the average age of people when they are diagnosed is 69 . About 6 of every 10 people diagnosed with stomach cancer each year are 65 or older [4]. When analyzing recent data concerning age distribution of gastric adenocarcinoma, we can note that age of onset of developing gastric cancer in Chinese and some African population is younger than that in the Western population [5]. Besides, we have also observed that the incidence rate for noncardia gastric cancer in the United States has declined among all race and age groups except for whites aged 25 to 39 years for whom it increases [6] [7].

In developed countries and developing countries, the incidence is higher in men than in women. Intestinal type is more common in males, 2:1 ratio worldwide. Diffuse-type males are equally distributed between males and females.

Concerning mortality rates, stomach cancer is the third leading cause of cancer death in both sexes worldwide ( 723,000 deaths, $8.8 \%$ of the total) [8] [9] [10]. The highest estimated mortality rates are in Eastern Asia (24 per 100,000 in men, 9.8 per 100,000 in women), the lowest in Northern America (2.8 and 1.5, respectively). High mortality rates are also present in both sexes in Central and Eastern Europe, and in Central and South America. This high mortality is probably explained by delays of diagnosis when the signs of complications appear. Countries like Japan who had adopted a screening program had successfully improved survival of gastric cancer [11]. So there is probably still the need to improve the 


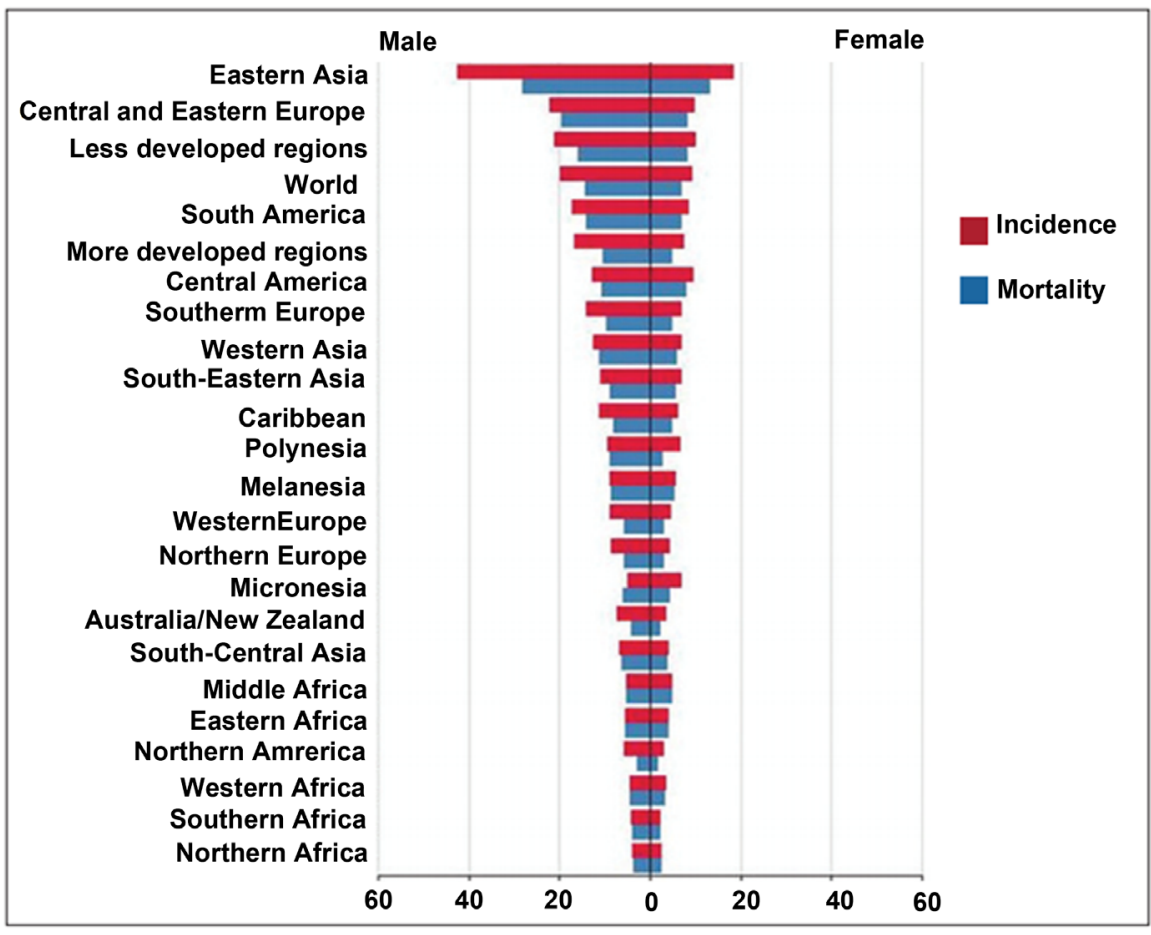

Figure 1. Graph shows the estimated cancer age-standardised incidence and mortality rates per 100,000 (Globocan. iarc 2012).

control of Helicobacter pylori infection and other dietary and environmental risk factors and to improve diagnosis and management of gastric cancer.

\subsubsection{Ethnicity}

In the US, Asian and Pacific Islanders have the highest incidence, followed by black, Hispanic, white, and American Indian [10]. Migration studies of patients born in high risk countries and moving to low risk countries shows that the risk is diminished but still significantly higher than those who are ethnically from a high risk country but born in a low risk country, indicating that early environmental exposure, rather than genetic factors, have a greater influence on risk [12] [13]. Racial and ethnic disparities in gastric cancer is a major public health concern and their recognition is necessary to develop interventions to eliminate these disparities. Some recent data suggests that factors such as tumor biology, environmental factors such as Helicobacter pylori infection and stage migration may probably explain such disparities [14].

\subsubsection{Time Trends: [3] [12] [13]}

Cancer incidence declines around the world at the rate of $2 \%$ to $3 \%$ per year. Considering that the world population is aging (especially in Japan), while the risk of cancer increases with age, the preventive effect is partially hidden from raw numbers but appears evident in the standardized age rates. Until the late 1930s, stomach cancer was the leading cause of cancer death in the United States. Now, stomach cancer is well down on this list. The decline of gastric cancer has interested both males and females. The decline of the incidence is related 
to lifestyle changes and the impact of environmental factors. In the US, this may be linked to increased use of refrigeration for food storage. Fruits and vegetables are becoming more available which decreased the use of salted and smoked foods. The frequent use of antibiotics to treat infections can kill the bacteria called Helicobacter pylori which are one of the major causes of stomach cancer (Figure 2).

The decline in the incidence does not concern all cancers of the stomach. In fact, this decrease primarily interested distal cancer while the cardia remains relatively stable or increases slightly with time. The incidence of intestinal type has declined rapidly over the recent few decades probably because it's more correlated to environmental factors and the infection by Helicobacter pylori. The incidence of diffuse type has declined more gradually which is more related to genetic factors. Regression of gastric cancer mortality depends not only on the incidence. It is influenced by advances in early detection of cancer in curable stage and premalignant lesions. This trend is particularly pronounced in Japan, where falling steadily since 1970 of gastric cancer mortality is explained by the widespread use of endoscopic and radiological screening.

\subsubsection{Other Descriptive Statistics: [4]}

For stomach cancer, death rates increase with age. The percent of stomach cancer deaths is highest among people aged 75 - 84 and the median age death in the US is 72 . There is also an improvement of the survival.

\section{Risk Factors of Gastric Cancer}

Gastric cancer is a multifactorial disease where the infection by the Helicobacter pylori bacteria plays the major role particularly for distal cancer. We can

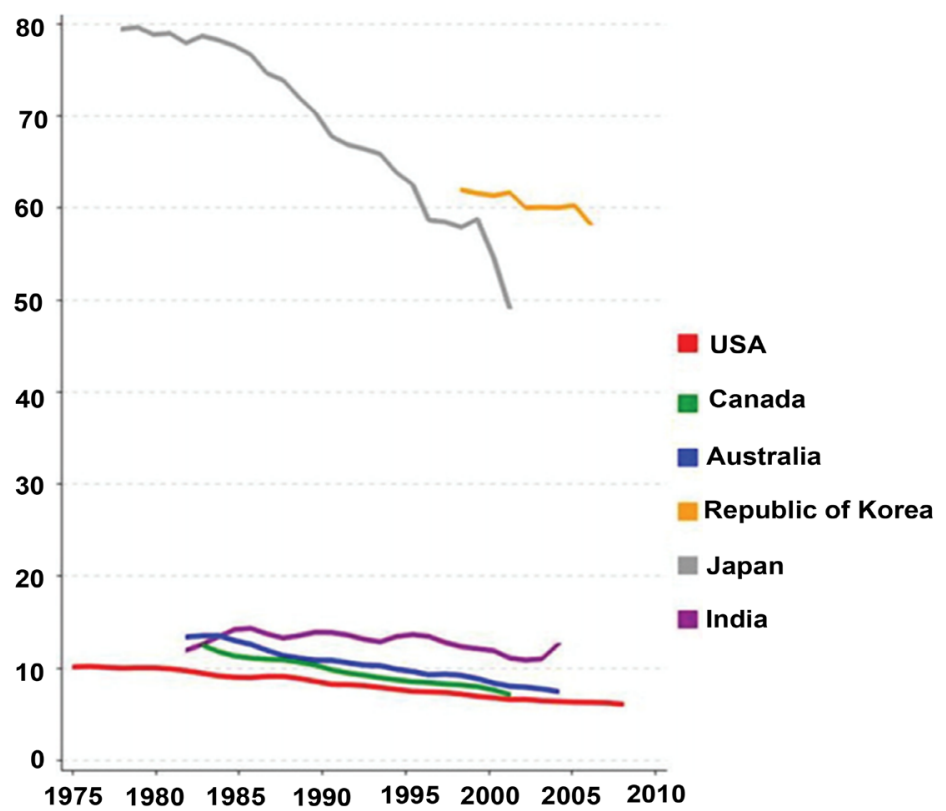

Figure 2. Graph shows the trends in Incidence of stomach cancer in selected countries : age-standardised rate per 100.00 men from Globocan IARC 2012. 
distinguish 4 categories of risk factors [13] [14] [15]: Helicobacter pylori infection, environmental factors, precancerous conditions and genetic predisposition. Major risk factors of gastric cancer are summarized in Table 1.

\subsection{Helicobacter pylori Infection}

H. pylori is a common type of bacteria that grows in the digestive tract and has a tendency to attack the stomach lining. It infects the stomachs of roughly 60 percent of the world's adult. This infection is an important and established risk factor of gastric cancer. This infection is the most frequent chronic infection in the world, it affects about $50 \%$ of the world's population but less than $1 \%$ of infected individuals will develop gastric cancer [16]. It has been clearly demonstrated that the prevalence of Helicobacter pylori infection varies between developed countries (less than $40 \%)$ and developing countries $(80 \%-90 \%)$ probably because of the socioeconomic conditions and hygiene. This bacterium was discovered in 1983 by Warren and Marshall (Nobel Prize in 2005) and it was not until 1994 that it was classified carcinogenic grade I. Current epidemiological evidence suggests that infection is usually acquired during childhood [16]. Although $H$. pylori infection is thus not a sufficient cause for the development of gastric cancer, it has been associated with high prevalence of gastric cancer and can also be found in the gastric mucosa of patients with chronic gastric inflammation [17]. Data from clinical trials have also suggested that $H$. pylori eradication therapy can effectively reduce the development of precancerous lesions and gastric cancer [18]. Guy D Eslick published in 2006, the result of 5 meta-analyses that have demonstrated the positive association between this infection and gastric cancer. The pooled estimates from this five meta analyses ranged between $1.92-2.65$ (mean 2.28) with confidence intervals ranging between 1.35 - 3.55. Studies have shown later that $H$. pylori $\mathrm{Cag}+, \mathrm{Vac}+$ type $\mathrm{s} 1$ and $\mathrm{m} 1$ is more aggressive and carcinogenic than the other types and the association is strongest [18].

Table 1. Summary of major risk factors of gastric cancer.

\begin{tabular}{cc}
\hline Major Risk factors for non-cardia gastric cancer & Major risk factors for cardia cancer \\
\hline Age & Age \\
Helicobacter pylori+++ & Male \\
Male & Tobacco smoking +/- Alcohol \\
Race & Race \\
Familial predisposition & Familial predisposition \\
Tobacco smoking & Overweight +++ \\
Overweight & ------ \\
Nitrites and nitrosamines & ------ \\
Salty and smoked food & Helicobacter pylori + \\
Low consumption of fibers and vitamin C & ------ \\
Previous gastrectomy & ----- \\
\hline
\end{tabular}


Many recent studies (randomized trials) demonstrated the interest of early eradication of infection in the prevention of gastric cancer. Fuccio et al. [19] published in 2009 a meta-analysis of 7 randomized trials that compared eradication treatment with no treatment in $\mathrm{H}$. pylori-positive patients and that assessed gastric cancer or progression of preneoplastic lesions during follow-up. Overall, 37 of 3388 (1.1\%) treated patients developed gastric cancer compared with 56 of 3307 (1.7\%) untreated (control) participants. The mechanism of carcinogenesis is not well known. H. pylori infection can cause chronic gastritis, this chronic condition can lead to gastric mucosal atrophy and intestinal metaplasia which are considered to be precancerous lesions of GC. In a pooled analysis of 6 studies with a total of 6695 participants followed from 4 to 10 years, the relative risk for gastric cancer was 0.65 (95\% CI, 0.43 to 0.98 ) [19] [20]. So he concluded that eradication of Helicobacter pylori infection lowers the risk of gastric cancer. One of the major steps to prevent gastric cancer is the identification of $H$. pylo$r i$-infected persons at high risk for gastric cancer, so that these individuals can be targeted by therapeutic interventions. Recent data have shown that $H$. pylori infection is characterized by a high level of interspecies genetic diversity. Comparing gastric cancer and non cancer gastric pathology related to $H$. pylori has demonstrated differences among $H$. pylori strains especially the presence or absence of a chromosomal region called the cag pathogenicity island (PAI) [21]. Current Knowledge shows that the risk of gastric cancer is very low among persons harboring $H$. pylori strains that lack the cag PAI. Among persons harboring strains that contain the cag PAI, the risk of gastric cancer is probably higher and is shaped by other host factors [21].

\subsection{Environmental Factors}

\section{- Tobacco:}

Smoking is a well-established risk factor for gastric cancer as many as different others cancers found to be associated with smoking in a cohort study of 34,439 British doctors over 50 years [16]. In a large population-based study in Europe (EPIC), $17.6 \%$ of gastric cancer cases were attributed to smoking. A pooled analysis of two population-based cohort studies in Japan has indicated that compared to those who never smoked, the relative risk for gastric cancer in current smokers was 1.84 and for past smokers 1.77 [22]. Smoking increases risk for both cardia and non-cardia cancers. It has also been demonstrated that the risk related to smoking is higher with alcohol consumption and is dose dependent. Risk diminishes with smoking cessation $>10$ years [22] [23].

- Alcohol

There is clearly inconsistent association between alcohol consumption and risk of gastric cancer mainly because of confounders in the majority of studies. However, A recent meta-analysis showed that alcohol consumption increases the risk of gastric cancer with an odds ratio (OR) of 1.39 (95\% CI 1.20 - 1.61) [24]. Besides, heavy drinking was assessed as a risk factor in the EPIC studies with a 
RR of 1.6 and 1.4 respectively [25] [26] [27].

- Tea and Coffee: [28]

There is no established association between these two elements and the risk of gastric cancer. A metaanalysis have recently shown that there was no association between coffee consumption and gastric cancer risk. However, high coffee intake (more than 6.5 cups/day) might increase the risk in the US population. There is probably a need for more studies to clarify the association.

- Diet:

a) Salt:

Many ecological, cohort and case control studies have confirmed the role of salt in gastric carcinogenesis and shown a positive association with gastric cancer [29]. The risk appears to be related to the dose of salt in foods. D'Elia published in 2012 [30] a meta-analysis of salt consumption and risk of gastric cancer. He found a pooled relative risk of 1.68 with a CI $1.17-2.41$. A confusion with nitrosamine steel remains in the majority of studies

b) N-nitroso compounds (NOCs):

Nitrite and nitrate are naturally occurring molecules in vegetables and also added to cured and processed meats to delay spoilage and pathogenic bacteria growth. Research over the past 15 years has led to a paradigm change in our ideas about health effects of both nitrite and nitrate. The epidemiological evidence linking gastric cancers in humans with nitrite and nitrate in the diet is lacking. A review of the epidemiological literature [31] found that the evidence was inconclusive especially when other factors like helicobacter pylori infection are strongly correlated to gastric cancer risk. An updated review in 2012 [32] and The Netherlands Cohort [33] came to the firm conclusion that there is no association, in humans, between nitrosamine formation and gastric cancer.

c) Meat and fat intake:

Higher intake of meat (red and processed meat) increases the risk of non-cardia gastric especially in subjects infected by Helicobacter pylori. The abundance of heme iron precursor of nitrosamine in red meat explains this increased risk. In the EPIC study this association was confirmed, for every 50 gr/day increase, the risk of non cardia gastric cancer increase by 2.45 -fold [31] [32].

d) fruit, vegetables and vitamin $\mathrm{C}$

Fruits and raw vegetables (allium vegetables) rich in vitamin $\mathrm{C}$ and anti-oxydant reduces the risk of gastric cancer according to a panel of cohort and case control studies. The recent studies and meta analyses however fairly demonstrated that the protective effect is weaker than the effect earlier described [34]. Vitamin C may be associated with a reduced risk of gastric cancer by inhibiting formation of $\mathrm{N}$-nitroso compounds. Larsson in 2006 published a meta-analysis with a protective effect with highest intake. Other micronutrients that have possible protective effects include zinc, vitamin E and carotenoids [34].

e) Fish intake: [28]

In a metanalysis published in 2011 [35], studies published between 1991 and 
2009 (15 case-control and 2 cohort studies) reported 5323 cases of gastric cancer and over 130,000 non-cases were included. The summary results from all studies indicated that the association between high fish consumption and reduced gastric cancer risk was not statistically insignificant $(\mathrm{RR}=0.87,95 \% \mathrm{CI}=0.71$ 1.07) [35]. Thus, the relation remains unclear till now.

f) obesity:

Obesity have been shown to be associated with a large number of cancers.A recent metaanalysis of the published cohort and case control studies indicated that obesity was associated with the risk of gastric cancer, especially for males and non-Asian populations particularly with cardia cancer [36].

\subsection{Other Risk Factors}

Epstein-Barr virus role in carcinogenesis is still unclear (EBV is detected in 5\% $15 \%$ of gastric cancers worldwide) [29]. Radiation exposure may also increase the risk of gastric cancer [37].

\subsection{Precancerous Conditions: [38] [39] [40] [41]}

These precancerous conditions include all host related factors and situations where the risk of gastric cancer is higher.

\subsubsection{Previous Gastric Surgery: Gastrectomy}

This situation happens especially after distal gastrectomy, the remnant stomach can be at high risk of adenocarcinoma [38]. The incidence of gastric stump carcinoma is estimated to be $1 \%-2 \%$, according to the current literature [39]. The risk appears after 20 - 30 years of surgery of benign disease especially for patients treated with Billroth II. This risk also increases with the persistence of $H$. pylori.

\subsubsection{Hypertrophic Gastropathy and Biermer Disease}

Menetrier disease (multiple sheet-like adenomas with associated foveol hyperplasia) is considered to be premalignant since $10 \%-15 \%$ of affected individuals develop gastric cancer. Biermer disease is defined as a macrocytic anemia caused by vitamin B12 deficiency, as a result of intrinsic factor deficiency. It is associated with atrophic body gastritis, whose diagnosis is based on histological confirmation of gastric body atrophy. In the literature, the annual incidence of gastric cancer ranges from $0.1 \%$ to $0.5 \%$.

\subsubsection{Gastric Polyps}

Those at risk of transformation to adenocarcinoma are fundic gland polyps associated with polyposis syndromes, hyperplastic polyps, particularly those $>2 \mathrm{~cm}$ have a small malignant potential and adenomatous polyps.

\subsection{Family History and Genetic Predisposition Factors: [39] [40] [41] [42]}

\subsubsection{Family History}

If Family history of gastric cancer in a relative in the first degree, the risk of gas- 
tric cancer is 2 - 3 times higher. In the case of 2 relatives, this risk is 10. In Case of gastric atrophy the OR is 2.20 with a CI $1.26-3.82$, and in case of metaplasia this risk is 1.98 with a CI $1.36-2.88$.

\subsubsection{Genetic Predisposition}

- Hereditary diffuse gastric cancer: This inherited syndrome greatly increases the risk of developing stomach cancer. The lifetime stomach cancer risk among affected people is about $70 \%$ to $80 \%$. It caused by mutations in the CDH1 gene.

- Hereditary non-polyposis colorectal cancer (HNPCC): It's an inherited genetic disorder that increases the risk of colorectal cancer. This disorder is caused by a defect in either the MLH1 or MSH2 gene, but other genes can cause HNPCC. The risk includes gastric cancer, urinary tract cancer etc.

- Familial adenomatous polyposis (FAP): This syndrome is characterized by multiple polyps in the colon stomach and small intestines. People carrying the APC mutation have a high risk of colorectal cancer and have a slightly increased risk of getting stomach cancer.

- BRCA1 and BRCA2: People who carry mutations of the inherited breast cancer genes BRCA1 or BRCA2 may also have a higher rate of stomach cancer.

- Li-Fraumeni syndrome/Peutz-Jeghers syndrome (PJS).

- Certain polymorphysm has been associated with gastric cancer: IL1-B/IFN gamma.

\section{Gastric Cancer Enigma}

The majority of epidemiological studies published in the recent years show a decline in the incidence of gastric cancer in the world. However, given the confusion between the distal and the cardiacancer that are often included in the property, there is no own statistics for each type of cancer in the world.This issue is a future challenge for researchers to establish the best preventive strategies. We also know that Asian, black and Hispanic people are at higher risk in comparison to other ethnic groups, but the real explanation for this disparities steel remains undetermined( genetic polymorphism? Environmental factors?)

Another enigma concerns the low frequency of gastric cancer in Africa even if the prevalence of helicobacter pylori is higher than in other countries (does it have a relation with genetic characteristics of the bacteria, with Host factors? Or environmental factors?) Besides, why is gastric cancer lower in patient with previous history of duodenal ulcer?

Currently, the development of science has enabled a better understanding of gastric cancer. In fact, there are several risk factors recognized in particular $\mathrm{He}$ licobacter pylori infection, smoking and genetic predisposing conditions. However, studies failed to answer a number of questions including the real role of alcohol in the distal gastric cancer? The reasons for male predominance in gastric cancer? The actual mechanisms by which all these factors cause the emer- 
gence of cancer cells in the gastric wall? Genetic predisposition syndromes are well described and the risk of gastric cancer exists and should be recognized to prevent invasive forms. What about the protective factors? The majority of studies failed to prove the protective role of tea in gastric cancer probably because of other carcinogenic confounders that exist in the majority of studies. Future studies also need to clarify the role of antioxidant, vitamin C and Cox 2 inhibitors in the chemoprevention of gastric cancer. Eradication of Helicobacter pylori is now recognized as the most effective preventive measure. It definitely reduces the incidence of gastric cancer. But how to diagnose this infection (endoscopy? Breath test? Serology?) Guidelines are different across the countries. Uniform strategy does not exist concerning endoscopic technics in screening and treatment of early gastric cancer. Long term Follow up need to be assessed in future studies to determine the role of the new endoscopic techniques (narrow band imaging, endomicroscopy, chromo endoscopy...).

\section{Conclusions}

Although incidence is decreasing according to recent statistics, gastric cancer remains the fifth cancer cause worldwide and the third leading cause of cancer death in both sexes. The 5 years survival does not exceed $30 \%$ and efforts to improve the treatment are disappointing. Best recognition of its risk factors, physiopathology is the key to prevent this cancer. Future studies should emphasize on:

- The protective factors and chemoprevention as reported previously.

- The investigation of the interaction between Helicobacter pylori infection and Host factors.

- Explore the interaction between environmental factors and genetic polymorphism.

- Explore biomarkers for gastric cancer and prognostic factors.

\section{Conflict of Interests}

All authors agree with the content of the manuscript and there are no conflicts of interests between them.

\section{References}

[1] Siegel, R., Ma, J., Zou, Z. and Jemal, A. (2014) Cancer Statistics. CA: A Cancer Journal for Clinicians, 64, 9. https://doi.org/10.3322/caac.21208

[2] Zhu, A.L. and Sonnenberg, A. (2012) Is Gastric Cancer again Rising? Journal of Clinical Gastroenterology, 46, 804. https://doi.org/10.1097/MCG.0b013e3182604254

[3] http://globocan.iarc.fr/old/FactSheets/cancers/stomach-new.asp

[4] http://seer.cancer.gov/statfacts/html/stomach.html

[5] Chan, A.O.O. and Wong, B. (2017) Epidemiology of Gastric Cancer.

[6] Colquhoun, A., Arnold, M., Ferlay, J., Goodman, K.J., Forman, D. and Soerjomataram, I. (2015) Global Patterns of Cardia and Non-Cardia Gastric Cancer Incidence 
in 2012. Gut, 64, 1881-1888. https://doi.org/10.1136/gutjnl-2014-308915

[7] Anderson, W.F., Camargo, M.C., Fraumeni Jr., J.F., et al. (2010) Age-Specific Trends in Incidence of Non Cardia Gastric Cancer in US Adults. JAMA, 303, 1723. https://doi.org/10.1001/jama.2010.496

[8] https://fcds.med.miami.edu/scripts/fcdspubrates/production/doSelection.aspx?selec tion=map

[9] http://www.cancer.org/

[10] http://apps.nccd.cdc.gov/uscs/toptencancers.aspx

[11] Ferro, A., Peleteiro, B., Malvezzi, M., Bosetti, C., Bertuccio, P., Levi, F., et al. (2014) Worldwide Trends in Gastric Cancer Mortality (1980-2011), with Predictions to 2015, and Incidence by Subtype. European Journal of Cancer, 50, 1330-1344. https://doi.org/10.1016/j.ejca.2014.01.029

[12] Fitzsimmons, D., Osmond, C., George, S. and Johnson, C.D. (2007) Trends in Stomach and Pancreatic Cancer Incidence and Mortality in England and Wales, 1951-2000. British Journal of Surgery, 94, 1162. https://doi.org/10.1002/bjs.5751

[13] Schlansky, B. and Sonnenberg, A. (2011) Epidemiology of Noncardia Gastric Adenocarcinoma in the United States. The American Journal of Gastroenterology, 106, 1978. https://doi.org/10.1038/ajg.2011.213

[14] Merchant, S.J., Li, L. and Kim, J. (2014) Racial and Ethnic Disparities in Gastric Cancer Outcomes: More Important than Surgical Technique? World Journal of Gastroenterology, 20, 11546-11551. https://doi.org/10.3748/wjg.v20.i33.11546

[15] Bendell, J. (2008) Latest Data on the Treatment of Upper Gastrointestinal Cancers. ASCO Education Book, 184-190.

[16] Cavaleiro-Pinto, M., Peleteiro, B., Lunet, N. and Barros, H. (2011) Helicobacter pylori Infection and Gastric Cardia Cancer: Systematic Review and Meta-Analysis. Cancer Causes Control, 22, 375-387. https://doi.org/10.1007/s10552-010-9707-2

[17] Eslick, G.-D. (2006) Helicobacter pylori Infection Causes Gastric Cancer? A Review of the Epidemiological, Meta-Analytic, and Experimental Evidence. World Journal of Gastroenterology, 12, 2991-2999.

[18] Khatoon, J., Rai, R.P. and Prasad, K.N. (2016) Role of Helicobacter pylori in Gastric Cancer: Updates. World Journal of Gastrointestinal Oncology, 8, 147-158. https://doi.org/10.4251/wjgo.v8.i2.147

[19] Fuccio, L., Zagari, R.M., Eusebi, L.H., et al. (2009) Meta-Analysis: Can Helicobacter pylori Eradication Treatment Reduce the Risk for Gastric Cancer? Annals of Internal Medicine, 151, 121-128. https://doi.org/10.7326/0003-4819-151-2-200907210-00009

[20] Lu, B. and Li, M. (2014) Helicobacter pylori Eradication for Preventing Gastric Cancer. World Journal of Gastroenterology, 20, 5660-5665. https://doi.org/10.3748/wjg.v20.i19.5660

[21] Timothy, L. and Covera, B.C. (2016) Helicobacter pylori Diversity and Gastric Cancer Risk. mBio, 7, e01869-15.

[22] Doll, R., Peto, R., Boreham, J. and Sutherland, I. (2005) Mortality from Cancer in Relation to Smoking: 50 Years Observations on British Doctors. British Journal of Cancer, 92, 426-429. https://doi.org/10.1038/sj.bjc.6602359

[23] Gonzalez, C.A., Pera, G., Aqudo, A., et al. (2003) Smoking and the Risk of Gastric Cancer in the European Prospective Investigation into Cancer and Nutrition (EPIC). International Journal of Cancer, 107, 629-634. https://doi.org/10.1002/ijc.11426 
[24] Koizumi, Y., Tsubono, Y., Nakaya, N., et al. (2004) Cigarette Smoking and the Risk of Gastric Cancer: A Pooled Analysis of Two Prospective Studies in Japan. International Journal of Cancer, 112, 1049-1055. https://doi.org/10.1002/ijc.20518

[25] Ma, K., Baloch, Z., He, T.-T. and Xia, X.S. (2017) Alcohol Consumption and Gastric Cancer Risk: A Meta-Analysis. Medical Science Monitor, 23, 238-246. https://doi.org/10.12659/MSM.899423

[26] Duell, E.J., Travier, N., Lujan-Barraso, L., et al. (2011) Alcohol Consumption and Gastric Cancer Risk in the European Prospective Investigation into Cancer and Nutrition (EPIC) Cohort. The American Journal of Clinical Nutrition, 94, 1266-1275. https://doi.org/10.3945/ajcn.111.012351

[27] Moy, K.A., Fan, Y., Wang, R., Gao, Y.T., Yu, M.C. and Yuan, J.M. (2010) Alcohol and Tobacco Use in Relation to Gastric Cancer: A Prospective Study of Men in Shanghai, China. Cancer Epidemiology, Biomarkers \& Prevention, 19, 2287-2297. https://doi.org/10.1158/1055-9965.EPI-10-0362

[28] Zeng, S.-B., Weng, H., Zhou, M., Duan, X.-L., Shen, X.-F. and Zeng, X.-T. (2015) Long-Term Coffee Consumption and Risk of Gastric Cancer: A PRISMA-Compliant Dose-Response Meta-Analysis of Prospective Cohort Studies. Medicine, 94, e1640. https://doi.org/10.1097/MD.0000000000001640

[29] Tsugane, S. (2005) Salt, Salted Food Intake, and Risk of Gastric Cancer: Epidemiologic Evidence. Cancer Science, 96, 1-6. https://doi.org/10.1111/j.1349-7006.2005.00006.x

[30] D’Elia, L., Rossi, G., Ippolito, R., Cappuccio, F.P. and Strazzullo, P. (2012) Habitual Salt Intake and Risk of Gastric Cancer: A Meta-Analysis of Prospective Studies. Clinical Nutrition, 31, 489-498. https://doi.org/10.1016/j.clnu.2012.01.003

[31] Jakszyn, P. and Gonzalez, CA. (2006) Nitrosamine and Related Food Intake and Gastric and Oesophageal Cancer Risk: A Systematic Review of the Epidemiological Evidence. World Journal of Gastroenterology, 12, 4296-4303. https://doi.org/10.3748/wjg.v12.i27.4296

[32] Bryan, N.S., Alexander, D.D., Coughlin, J.R., Milkowski, A.L. and Boffetta, P. (2012) Ingested Nitrate and Nitrite and Stomach Cancer Risk: An Updated Review. Food and Chemical Toxicology, 50, 3646-3665. https://doi.org/10.1016/j.fct.2012.07.062

[33] Keszei, A.P., Goldbohm, R.A., Schouten, L.J., Jakszyn, P. and van den Brandt, P.A. (2013) Dietary N-Nitroso Compounds, Endogenous Nitrosation, and the Risk of Esophageal and Gastric Cancer Subtypes in the Netherlands Cohort Study. The American Journal of Clinical Nutrition, 97, 135-146. https://doi.org/10.3945/ajcn.112.043885

[34] González, C.A., Pera, G., Aqudo, A., et al. (2006) Fruit and Vegetable Intake and the Risk of Stomach and Oesophagus Adenocarcinoma in the European Prospective Investigation into Cancer and Nutrition (EPIC-EURGAST). International Journal of Cancer, 118, 2559-2566. https://doi.org/10.1002/ijc.21678

[35] Wu, S., Liang, J., Zhang, L., Zhu, X., Liu, X. and Miao, D. (2011) Fish Consumption and the Risk of Gastric Cancer: Systematic Review and Meta-Analysis. BMC Cancer, 11, 26. https://doi.org/10.1186/1471-2407-11-26

[36] Lin, X.J., Wang, C.P., Liu, X.D., Yan, K.K., Li, S., Bao, H.H., Zhao, L.Y. and Liu, X. (2014) Body Mass Index and Risk of Gastric Cancer: A Meta-Analysis. Japanese Journal of Clinical Oncology, 44, 783-791. https://doi.org/10.1093/jico/hyu082

[37] Murphy, G., Pfeiffer, R., Camargo, M.C. and Rabkin, C.S. (2009) Meta-Analysis Shows That Prevalence of Epstein-Barr Virus-Positive Gastric Cancer Differs Based on Sex and Anatomic Location. Gastroenterology, 137, 824-833. 
https://doi.org/10.1053/j.gastro.2009.05.001

[38] Malekzadeh, R., Derakhshan, M.H. and Malekzadeh, Z. (2009) Gastric Cancer in Iran: Epidemiology and Risk Factors. Archives of Iranian Medicine, 12, 576-583.

[39] Zhao, L., Blot, W.J., Liu, W.D., et al. (1994) Familial Predisposition to Precancerous Gastric Lesions in a High-Risk Area of China. Cancer Epidemiology, Biomarkers \& Prevention, 3, 461.

[40] Hemminki, K., Sundquist, J. and Ji, J. (2007) Familial Risk for Gastric Carcinoma: An Updated Study from Sweden. British Journal of Cancer, 96, 1272. https://doi.org/10.1038/sj.bjc.6603722

[41] Ballester, V. and Cruz-Correa, M. (2014) Endoscopic Surveillance of Gastrointestinal Premalignant Lesions: Current Knowledge and Future Directions. Current Opinion in Gastroenterology, 30, 477-483. https://doi.org/10.1097/MOG.0000000000000090

[42] Bevan, S. and Houlston, R.S. (1999) Genetic Predisposition to Gastric Cancer. QJM: An International Journal of Medicine, 92, 5-10.

https://doi.org/10.1093/qjmed/92.1.5 